\title{
Nano-Bio Interactions: Guiding the Development of Nanoparticle Therapeutics, Diagnostics, and Imaging Agents
}

\author{
Simon R. Corrie ${ }^{1,2} \cdot$ Kristofer J. Thurecht ${ }^{3,4}$
}

Received: 26 July 2016 / Accepted: 27 July 2016 / Published online: 8 August 2016

(C) Springer Science+Business Media New York 2016

Advances in nanotechnology are set to make a tremendous impact in medicine and biomedical research. Materials that are nano-sized or contain nano-sized domains possess unique optical, electrical and biological properties, which are typically absent in the bulk materials. In the emerging field of "nanomedicine", one of the biggest challenges facing researchers, clinicians, companies and regulators alike, is to improve our understanding of how nanomaterials interact with biological systems, and then to use this information to modulate the nano-bio interface. This requires a hierarchical approach, investigating interactions that occur at the level of molecules, cells, tissues, and ultimately entire organisms. In terms of medical applications, the use of nanoparticles can be grouped into diagnostics, therapeutics, and imaging agents. Diagnostic nanoparticles are generally designed to transduce and/or amplify a semi-quantitative signal resulting from a biosensor or bioassay, designed for either in vitro or in vivo use. Common examples would include the coloured latex nanoparticles used for signal amplification in lateral flow immunoassays, however advances in technology have resulted in the development of nanosensors that can be deployed in vivo for real-time biomolecular sensing. For imaging applications, the goal is to design materials that provide sufficient contrast for the myr-

Simon R. Corrie

simon.corrie@monash.edu

Department of Chemical Engineering, Monash University, Clayton, VIC, Australia 3800

2 ARC Centre of Excellence in Convergent Bio-Nano Science and Technology, Monash University, Clayton, VIC, Australia 3800

3 Centre for Advanced Imaging, University of Queensland, St Lucia, QLD, Australia 4072

4 ARC Centre of Excellence in Convergent Bio-Nano Science and Technology, University of Queensland, St Lucia, QLD, Australia 4072 iad of imaging modalities utilised in nanomedicine (e.g., MR/PET/CT/ultrasound) in order to identify pathological features with high resolution. Arguably the most successful area to date has been in the development of nanotherapeutics (combinations of drugs and nanoparticles), which has resulted in $\sim 50$ FDA-approved products, with a further $\sim 75$ currently in clinical trials. The review by Bobo et al. provides an up-to-date snapshot of the most recent developments in this field, and provides an overview of each of the classes of nanomedicines under investigation to date. The other publications in this theme include reviews on emerging materials, along with primary articles providing fundamental insights into how new materials behave in applications ranging across the diagnostic, imaging and therapeutic areas.

The reviews submitted by the groups of Lee, Nann and Voelcker, provide in-depth summaries of emerging nanomaterials for biomedical applications. Li et al. focused on polymeric materials for $\mathrm{pH}$-sensitive imaging and drug delivery. An interesting focus here is on multi-modal systems, in which fluorescence and MR imaging is combined to take advantage of the unique aspects of each modality. Furthermore, the combination of $\mathrm{pH}$ - and temperaturesensitive materials is highlighted as an area for future emphasis, based on the demonstrated success of several in vivo studies. So far, most applications in this field have focused on cancer imaging and treatment, however as the authors state, many pathologies are characterized by unique $\mathrm{pH}$ and/or temperature profiles. Schroeder et al. reviewed the state-of-the-art in graphene quantum dots (GQDs), which have many similar characteristics with the more common inorganic quantum dots, but are easier to functionalize for aqueous systems and may exhibit lower intrinsic toxicity. The harsh synthetic strategies that are employed lead to a high density of accessible oxidized groups to allow chemical coupling to labels, targeting moieties or drugs. Importantly, the authors highlight that these materials also have intrinsic 
luminescence, however their fluorescent quantum yields are still relatively low, with spectral data dominated by size, purity and synthesis conditions. Aisyiyah Jenie et al. focused on luminescence-based sensors, with emphasis on inorganic structures and porous silicon materials in particular. In this space, research efforts are geared towards enhancing the detection limits of luminescent molecules and fluorophores by improving the fluorescence lifetime and quantum yield. Combined with this, the authors suggested that the combination of enhanced luminescence with porous silicon devices could result in ultra-sensitive optical biosensors for both in vitro and in vivo applications. In combination, these reviews provide a broad account of several interesting materials, along with a discussion of common issues faced by researchers in this field, including understanding how the materials interact with biological systems, and how to evaluate their subsequent behavior.

Moving to the primary articles in the theme, we start with the development of tools to understand how nanoparticles interact with cells. Mann et al. demonstrated the use of a DNA hybridization sensor to measure how many nanoparticles are internalised by certain cells, as opposed to simply being associated with the cell membrane. This work tackles a key challenge in nanomedicine research; definitively showing the difference between cell-membrane association versus internalization, especially when using traditional microscopy approaches. The authors used their "SHIP" probe in conjunction with inhibition studies to investigate the mechanism of uptake for a polymeric nanoparticle for two very different cell lines. These sensors and others will likely be developed to help map the pathways of cellular internalization of nanoparticles, to allow for controlled drug release in specific sub-cellular compartments.

Pearce et al. investigated the accumulation of polymeric nanomedicines in prostate tumor cells, using targeted and non-targeted approaches. They showed that by targeting two different cell-surface receptors on different cell lines, they could enhance the accumulation of the nanomedicines in these cells, both in vitro and in vivo (subcutaneous tumor models in mice). The authors utilised multispectral optical imaging to monitor the accumulation of these materials within tumor tissue, and also to semiquantitatively analyse the levels of non-specific accumulation in non-target tissues. Interestingly, the authors caution that increased accumulation does not necessarily lead to enhanced efficacy, because accumulation does not alone indicate cellular internalization or drug distribution. However, the enhanced accumulation achieved with targeting implies that these particles are binding to target cells of interest, rather than simply accumulating within the tumour microenvironment as a result of the so-called "EPR" effect. This approach can also be used to identify likely clearance mechanisms, given that there was a difference observed in the ratio of kidney/liver accumulation for the two nanomedicines.

Moving to the imaging arena, Woodard et al. designed and synthesised a targeted imaging agent for application in monitoring atherosclerotic plaques, evaluating biodistribution, pharmacokinetics and efficacy in a mouse model. Based on their novel polymeric "comb" nanoparticles developed previously in Hawker's group, they were able to combine several functional monomers into the material - including PEG (anti-fouling), ${ }^{64} \mathrm{Cu}$ (PET agent), and a targeting agent (CANF; c-atrial natiuretic peptide) that binds to a known receptor that was previously shown to be a good biomarker for plaques in animal and human coronary arteries. The biodistribution profile of the nanoparticles in C57 mice changed significantly with the addition of targeting agent, with reduced accumulation in the liver and spleen in comparison to non-targeted materials. By titrating the CANF-monomer in the particle synthesis reactions, the authors showed that the PET signal generated in the aortic arch was significantly enhanced in the disease model.

In the diagnostics area, Clark's group have developed a number of nanoparticle-based sensors with potential for real-time measurement of biological processes in animals. In particular, they have demonstrated in vivo monitoring of ions and small molecules; this is an exciting and evolving area of nanomedicine research. In this study, Di et al. fabricated electrospun fibres embedded with $\mathrm{pH}$ sensors for monitoring of 3D cell cultures. These cultures (or spheroids) are important model systems for drug screening etc., and real-time monitoring of differences in biochemical analytes throughout the spheroid can provide key information on the health of the cells and the potential effects of drug candidates. The authors compared PCL and PLGA fibre systems, and found that the PCL yielded a more suitable dynamic range and sensitivity for $\mathrm{pH}$ sensing. This was related to the degradation behaviour of the materials in biological systems, hence the embedded "optodes" provide spatio-temporal information concerning both the cell biochemistry and the material properties. Sensors based on this concept could find application not only in biosensing, but in imaging, tissue engineering, and other therapeutic applications where a sensing event would be beneficial. 
Bioassays, as opposed to biosensors, are an enduring component of biomedical and clinical laboratories, however there is much research geared towards increasing the sensitivity and reducing detection limits to facilitate rare biomarker detection and analysis. Lai's group has introduced a unique concept that involves capture of analytes onto magnetic nanoparticles followed by aggregation of particles using temperaturesensitive pNIPAAM polymer to facilitate rapid magnetophoretic separation at high efficiencies. In this particular study, Phan et al. demonstrate that a recombinant pNIPAAM-CVN polymer/protein conjugate can be used to selectively bind the gp 120 protein on the surface of HIV. With the addition of pNIPAAM-modified magnetic nanoparticles and a temperature increase to $42^{\circ} \mathrm{C}$, the nanoparticles, and the pNIPAAM-CVN conjugate bound to HIV virions, aggregate to form $\sim 1 \mu \mathrm{m}$ particles which can be magnetically separated at a much faster rate than for the unmodified $\sim 10 \mathrm{~nm}$ magnetic nanoparticles. These enrichment protocols could be easily coupled with downstream detection systems to produce a highly sensitive device for rapid detection of rare biomarkers.

In order to fully realise the potential of nanotechnology in clinical applications, a number of significant hurdles must still be overcome; these include, but are not limited to, nonspecific protein adsorption which leads to aggregation and/or immune responses to nanomaterials, determining and controlling the biological fate of nanomaterials, as well as issues associated with scale-up and processing. These can be addressed through advances in fundamental science, translation of materials from research labs into clinical trials, and then ensuring that regulatory authorities have the information they need to ensure that marketed products are safe and efficacious. While nanomedicines certainly are making headway into both clinical trials and mainstream application, the complexity of the biological-nanomaterial interface means that future development of more advanced systems must address these concerns. The evolution in understanding of such processes, including for example, the formation of the protein corona on nanoparticles, means that next generation nanomedicines will most likely be designed to take advantage of such a phenomenon, rather than limit or minimize its presence. This exemplifies the importance of establishing multidisciplinary teams for evaluating nanomedicines. As biotechnology and nanotechnology continue to merge across disciplines, we envisage that exciting new advances will certainly ensue, with a concomitant increase in regulatory approval of nanomedicines.

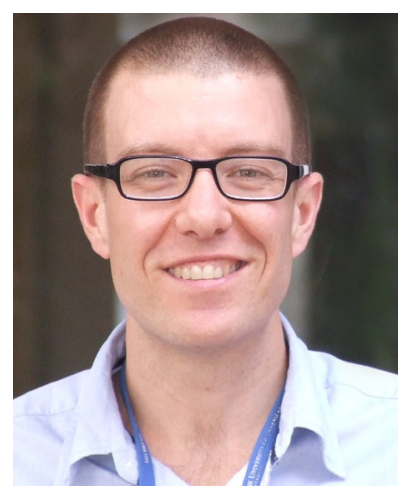

Dr Simon Gorrie is a Senior Lecturer in the Chemical Engineering Department at Monash University and is a chief investigator in the ARG Centre of Excellence in Convergent BioNano Science and Technology. He completed both his undergraduate degree in Chemical Engineering and his $\mathrm{PhD}$ in Chemistry at UQ, before undertaking postdoctoral studies at the HPV Research Laboratory at the University of Washington, Seattle, USA (American Australian Association Fellow), and at the Australian Institute for Bioengineering and Nanotechnology where he was an Associate Group Leader (as a QLD Smart Futures Fellow, and then subsequently as an ARC DECRA Fellow). He recently established the Nanosensor Engineering Laboratory at Monash University, a group focussed on developing clinically relevant biosensors and bioassays using a combination of material science and molecular biology.

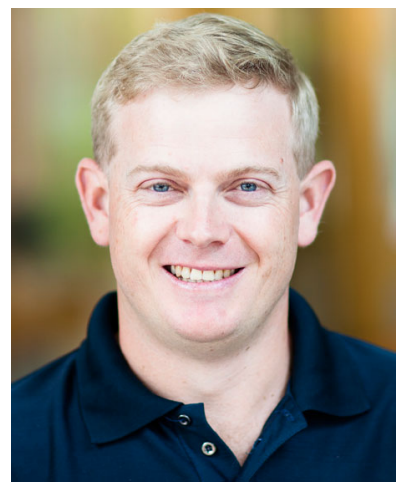

Assoc. Prof. Kristofer Thurecht graduated from the University of Queensland, Australia, in 2005 with a $\mathrm{PhD}$ in polymer chemistry. He is a Group Leader in the Centre for Advanced Imaging (CAI) and an Associate Group Leader at the Australian Institute for Bioengineering and Nanotechnology (AIBN) at the University of Queensland, where he is an ARC Future Fellow and a chief investigator in the ARC Centre of Excellence in Convergent Bio-Nano Science and Technology. He undertook postdoctoral studies in the UK working on polymer synthesis in supercritical CO2 with Prof. Steve Howdle at Nottingham University. In 2007, Dr Thurecht was simultaneously awarded a Ramsay Centenary Fellowship and 1851 Research Fellowship in the UK, and in the following year an ARC Australian Postdoctoral Fellowship. His current interests lie in developing polymeric nanomedicines and utilizing advanced molecular imaging techniques to understand their behaviour under physiological conditions. 